\title{
Constraining C III] Emission in a Sample of Five Luminous $z=5.7$ Galaxies
}

\author{
Jiani Ding ${ }^{1}$, Zheng Cai ${ }^{2,7}$, Xiaohui Fan ${ }^{1}$, Daniel P. Stark ${ }^{1}$, Fuyan Bian ${ }^{3}$, Linhua Jiang ${ }^{4}$, Ian D. McGreer ${ }^{1}$, \\ Brant E. Robertson ${ }^{5}$, and Brian Siana ${ }^{6}$ \\ ${ }^{1}$ Steward Observatory, University of Arizona, 933 N Cherry Avenue, Tucson, AZ, 85721, USA \\ ${ }^{2}$ UCO/Lick Observatory, University of California, 1156 High Street, Santa Cruz, CA, 95064, USA; zcai@ucolick.org \\ ${ }^{3}$ Research School of Astronomy and Astrophysics, The Australian National University, Cotter Road, Weston Creek, ACT 2611, Australia \\ ${ }^{4}$ Kavli Institute for Astronomy and Astrophysics, Peking University, Beijing 100871, China \\ ${ }^{5}$ Department of Astronomy and Astrophysics, University of California, Santa Cruz, Santa Cruz, CA 95064, USA \\ ${ }^{6}$ Department of Physics and Astronomy, University of California, Riverside, 900 University Avenue, Riverside, CA, 92521, USA \\ Received 2016 November 29; revised 2017 February 28; accepted 2017 March 3; published 2017 April 4
}

\begin{abstract}
Recent observations have suggested that the C III] $\lambda 1907 / 1909$ emission lines could be alternative diagnostic lines for galaxies in the reionization epoch. We use the F128N narrowband filter on the Hubble Space Telescope's (HST) Wide Field Camera 3 (WFC3) to search for C III] emission in a sample of five galaxies at $z=5.7$ in the Subaru Deep Field and the Subaru/XMM-Newton Deep Field. Using the F128N narrowband imaging, together with the broadband imaging, we do not detect C III] emission for the five galaxies with $J_{\mathrm{AB}}$ ranging from 24.10 to 27.00 in our sample. For the brightest galaxy J132416.13+274411.6 in our sample $\left(z=5.70, J_{\mathrm{AB}}=24.10\right)$, which has a significantly higher signal to noise, we report a C III] flux of $3.34 \pm 1.81 \times 10^{-18} \mathrm{erg} \mathrm{s}^{-1} \mathrm{~cm}^{-2}$, which places a stringent $3 \sigma$ upper limit of $5.43 \times 10^{-18} \mathrm{erg} \mathrm{s}^{-1} \mathrm{~cm}^{-2}$ on $\mathrm{C} \mathrm{III]} \mathrm{flux} \mathrm{and} 6.57 \AA$ on the C III] equivalent width. Using the stacked image, we put a $3 \sigma$ upper limit on the mean $\mathrm{C} \mathrm{III]} \mathrm{flux} \mathrm{of} 2.55 \times 10^{-18} \mathrm{erg} \mathrm{s}^{-1} \mathrm{~cm}^{-2}$ and a $3 \sigma$ upper limit on the mean C III] equivalent width of $4.20 \AA$ for this sample of galaxies at $z=5.70$. Combined with strong $\mathrm{C}$ III] detection reported among high- $z$ galaxies in the literature, our observations suggest that the equivalent widths of $\mathrm{C}$ III] from galaxies at $z>5.70$ exhibit a wide range of distribution. Our strong limits on C III] emission could be used as a guide for future observations in the reionization epoch.
\end{abstract}

Key words: dark ages, reionization, first stars - galaxies: high-redshift

\section{Introduction}

In recent years, we have witnessed great progress in studying galaxies at $z>6$. Several deep field surveys using the Hubble Space Telescope (HST) and largest ground-based telescopes (Ellis et al. 2013; McLure et al. 2013; Bouwens et al. 2015; Finkelstein et al. 2015; for a review, see Stark 2016) have given rise to hundreds of $z>6$ galaxy candidates. One of the main challenges for redshift confirmation in $z>6$ galaxy candidates is the increasing attenuation of $\operatorname{Ly} \alpha$ emission due to a rising intergalactic medium neutral fraction in the end of the reionization epoch (Caruana et al. 2014; Choudhury et al. 2015; Mesinger et al. 2015; Planck Collaboration et al. 2016). As a result, the efficiency and validity of using Ly $\alpha$ emission to study $z>6$ galaxies are limited, leading to the search for an alternative diagnostic line. Spectroscopic surveys among starforming galaxies at $z \sim 2$ (Stark et al. 2014) and $z \sim 7$ (Stark et al. 2015, 2017) reveal that C III] $\lambda 1907 / 1909$ could be used as alternative emission lines for determining the redshifts for high $z$ galaxies.

The strength of various nebular emission lines, such as He II $\lambda 1640$, C IV $\lambda 1549$, O III] $\lambda 1661 / 1667$, and C III] $\lambda 1907 / 1909$, can help to constrain galaxy physical properties, including the ionization parameters and metallicities (e.g., Erb et al. 2010; Cai et al. 2011). Recently, several efforts have been conducted to investigate the $\mathrm{C}$ III] emission from high- $z$ galaxies (e.g., Stark et al. 2014, 2015, 2017; Rigby et al. 2015; Zitrin et al. 2015). The C III] emission has been detected in a sample of Lyman break galaxies (LBGs) at $z \sim 3$ (Shapley et al. 2003) and in a sample of low-mass, low-luminosity star-forming

\footnotetext{
Hubble Fellow.
}

galaxies at $z \sim 2$ (Stark et al. 2014). Previous studies (Stark et al. 2014; Jaskot \& Ravindranath 2016) suggest that galaxies with strong $\mathrm{C}$ III] emission should have high ionization parameters $(\log U$ varies from -2.16 to -1.84$)$, low metallicities $\left(0.04-0.13 Z_{\odot}\right)$, and younger stellar populations (6-50 Myr) (detailed ranges from Stark et al. 2014). The photoionization models from Jaskot \& Ravindranath (2016) support that galaxies with strong $\mathrm{C}$ III] emissions may have $\log U \geqslant-2$ and metallicity $Z \sim 0.14 Z_{\odot}$ after taking into account the decline of $\mathrm{C} / \mathrm{O}$ ratio with decreasing metallicity. Such conditions may be common in typical $z \gtrsim 6$ galaxies (Stark et al. 2014; Jaskot \& Ravindranath 2016). Stark et al. (2015, 2017) conducted a pilot survey on the C III] emissions for $z \gtrsim 6-7$ galaxies, which are expected to have strong [O III] and $\mathrm{H} \beta$ emission from the rest-frame optical photometric measurements. They successfully detected robust C III] emission lines in one galaxy. Nevertheless, the current sample sizes in terms of galaxy numbers and galaxy properties are still too small for conducting a robust statistical study on C III] properties at $z \gtrsim 6$.

To better understand the fraction of galaxies that host strong $\mathrm{C}$ III] lines in the reionization epoch, it is crucial to conduct a systematic study to search for C III] emission in a statistical sample of high-redshift galaxies. There have been $>100$ highredshift Ly $\alpha$ emitters discovered at $z \sim 5.7$. The most extensive surveys are those using deep imaging in the Subaru Deep Field (SDF; Kashikawa et al. 2011) and Subaru/XMMNewton Deep Field (SXDF; Ouchi et al. 2005). The SDF field contains the brightest Ly $\alpha$ emitter (LAE), J132416.13 +274411.6 , at $z=5.70$ (Shimasaku et al. 2006), and the SXDF field contains an overdensity of LAEs at $z \approx 5.70$ 
Table 1

Photometry Results for Five Galaxies in Our Sample

\begin{tabular}{|c|c|c|c|c|c|}
\hline No. & $\begin{array}{c}\text { R.A. } \\
\text { (J2000.0) }\end{array}$ & $\begin{array}{c}\text { Decl. } \\
\text { (J2000.0) }\end{array}$ & $\begin{array}{l}\text { F128N Flux Density } \\
\left(10^{-20} \mathrm{erg} \mathrm{s}^{-1} \mathrm{~cm}^{-2} \AA^{-1}\right)\end{array}$ & $\begin{array}{l}\text { F125W Flux Density } \\
\left(10^{-20} \mathrm{erg} \mathrm{s}^{-1} \mathrm{~cm}^{-2} \AA^{-1}\right)\end{array}$ & $\begin{array}{l}\text { C III] Line Fluxes } \\
\left(10^{-18} \mathrm{erg} \mathrm{s}^{-1} \mathrm{~cm}^{-2}\right)\end{array}$ \\
\hline 1 & $13: 24: 16.13$ & $27: 44: 11.62$ & $14.30 \pm 1.10$ & $12.20 \pm 0.30$ & $3.34 \pm 1.81$ \\
\hline 2 & 02:17:47.32 & $-05: 26: 48.0$ & $10.60 \pm 0.70$ & $11.30 \pm 0.10$ & $-1.11 \pm 1.12$ \\
\hline 3 & $02: 17: 45.03$ & $-05: 28: 42.5$ & $3.50 \pm 0.68$ & $3.28 \pm 0.12$ & $0.35 \pm 1.10$ \\
\hline 4 & 02:17:50.00 & $-05: 27: 08.2$ & $1.42 \pm 0.64$ & $2.31 \pm 0.13$ & $-1.42 \pm 1.04$ \\
\hline 5 & 02:17:49.13 & $-05: 28: 54.2$ & $2.19 \pm 0.81$ & $2.00 \pm 0.15$ & $0.30 \pm 1.31$ \\
\hline Stacking & $\ldots$ & $\ldots$ & $8.64 \pm 0.53$ & $8.96 \pm 0.08$ & $-0.51 \pm 0.85$ \\
\hline
\end{tabular}

Note. The coefficients of the aperture for source $1-5$ is a kron factor of 1.8 and a minimum aperture of 2.5 semimajor radius. The F125W flux of source 1 is from the UV-continuum fitted by photometry results in F110W and F160W.

(Ouchi et al. 2005). Furthermore, deep HST observations in $Y$, $J$, and $H$ bands have been conducted to precisely measure continuum levels and rest-frame ultraviolet (UV) morphologies of the LAEs in these two fields (Jiang et al. 2013). The LAEs at $z \approx 5.7$ in SDF and SXDF are ideal for conducting a systematic study of C III] emission.

In this Letter, we present our deep, high-resolution narrowband $(\mathrm{F} 128 \mathrm{~N})$ and broadband imaging $(\mathrm{F} 125 \mathrm{~W})$ in SDF and SXDF fields using the HST Wide Field Camera 3 (WFC3) imaging. At $z=5.7$, the C III] emission is redshifted to the the most sensitive part of the F128 narrowband filter. Our HST observations targeted five $z=5.7$ galaxies in C III] emission using two $H S T$ pointings. The high sensitivity and low sky background with WFC3 allow us to probe C III] lines in LAEs at a level that is difficult to achieve from the ground. In this Letter, we report a tentative $2 \sigma$ flux excess in the F128N filter for the brightest LAE at $z=5.7$ in the SDF field. For the other four galaxies in the SXDF protocluster, we report a null detection of C III] emission. Using this sample of galaxies, we place a stringent upper limit on the $\mathrm{C}$ III] fluxes and equivalent widths (EWs) for the five galaxies in our sample at $z=5.7$. Organization of this Letter is as follows. In Section 2, we discuss our HST observation and data reduction. In Section 3, we measure the UV-slope, continuum, and morphology for the brightest galaxy in SDF and report $3 \sigma$ upper limits for C III] $\lambda 1907 / 1909$ emission line fluxes in the other four galaxies in the SXDF. In Section 4, we discuss the astrophysical implications of our photometry results. Throughout the whole Letter, we adopt a flat lambda-CDM cosmology with $\Omega_{\lambda}=0.7, \Omega_{\mathrm{m}}=0.3$, and $H_{0}=70 \mathrm{~km} \mathrm{~s}^{-1} \mathrm{Mpc}^{-1}$.

\section{Observation and Data Reduction}

We conduct deep HST/WFC3 narrowband $(\mathrm{F} 128 \mathrm{~N})$ and broadband (F125W) imaging in a sample of five galaxies. These galaxies are LAEs at $z=5.7$ selected from the SDF (Shimasaku et al. 2006; Kashikawa et al. 2011) and the SXDF (Ouchi et al. 2005). The galaxies described in this Letter are listed in Table 1. The redshift range of our sample is $z=5.69-5.75$. The redshifts are determined based on their Ly $\alpha$ emission. From Erb et al. (2014), the average Ly $\alpha$ velocity offset of LAEs with the absolute magnitudes of $M_{\mathrm{UV}} \leqslant-19.3$ (similar to that of our sample) at $z \sim 2-3$ is $\approx 300 \mathrm{~km} \mathrm{~s}^{-1}$. Assuming the Ly $\alpha$ systematic velocity offset for our LAE sample is $300 \mathrm{~km} \mathrm{~s}^{-1}$, the $\mathrm{C}$ III] $\lambda 1907 / 1909$ emission of our sample galaxies resides at 12745.1-12872.9 $\AA$, securely lying on most of the sensitive wavelengths of the F128N filter $(\lambda c=12832 \AA$, FWHM $=159 \AA)$ we used.
In Cycle 22, we use 14 orbits to conduct the HST/WFC3 observations in both SDF and SXDF fields. To detect the C III] line for brightest LAE at $z=5.7, \mathrm{~J} 132416.13+274411.6$ (source 1; see Table 1), we use six-orbit ( 16,000 s integration time) F128N imaging in the SDF field. Deep HST F110W and F160W broadband imaging of the brightest galaxy (source 1) have already been conducted in Jiang et al. (2013), and we use the F110W and F160W imaging to determine the continuum level and continuum slope of source 1 . We assign another six-orbit $(\sim 16,000 \mathrm{~s}$ integration time) F128N imaging for the other four galaxies at $z=5.7$ in the SXDF (sources 2-5; see Table 1). To conduct the accurate continuum subtraction for our galaxies in the SXDF, we use two-orbit (5223.5 s integration time) F125W imaging for sources $2-5$ in the SXDF. These observations allow us to reach an F128N depth of $1-\sigma=1 \times 10^{-18} \mathrm{erg} \mathrm{s}^{-1} \mathrm{~cm}^{-2}$ in the SDF and SXDF fields, enabling the detection of $\mathrm{C}$ III] emission as weak as $\sim 6 \AA$ at the $3 \sigma$ level for the brightest target in our sample.

We distribute our entire observations into four individual visits. For each visit, a standard four-point dither sequence is applied to populate each of the two orbits. The data reduction is conducted using Multidrizzle (Koekemoer et al. 2003), and the detailed procedures follow the descriptions in Cai et al. (2011, 2015). To optimize the output data quality, we choose a final output pixel scale of 0 " 06 instead of the initial pixel scale 0 ". 13 and final pixfac parameter 0.7 (shrinking pixel area) after different trials of combinations of parameters. The final output images we obtained from different $H S T$ band imaging for source 1 are shown in Figure 1 and sources 2-5 in Figure 2.

\section{Measured C III] Fluxes and Upper Limits}

Similar to Cai et al. (2011, 2015), we use SExtractor (Bertin $\&$ Arnouts 1996) to measure the fluxes in the narrowband (F128N) and broadband filters (F110W and F160W) for our galaxy sample. For sources $1-5$, we apply an $M_{A} G_{A U T O}$ elliptical aperture with a kron factor of 1.8 and a minimum aperture of 2.5 semimajor radius to measure the flux in the narrowband imaging. We apply the same aperture to the broadband imaging (Figures 1 and 2).

The photometry for source 1 is presented in Table 1 and Figure 3. We use our photometry results in the F110W broadband and F160W broadband to fit the spectral energy distribution, assuming a standard power-law continuum $f_{\mathrm{C}}=\alpha(\lambda / 10000 \AA)^{\beta}$, where $\alpha$ is a constant. We find $f_{\mathrm{C}}(\lambda)=1.82 \pm 0.10 \times 10^{-19}(\lambda / 10000 \AA)^{-1.60 \pm 0.20} \mathrm{erg} \mathrm{s}^{-1}$ $\mathrm{cm}^{-2} \AA^{-1}$. The F128N filter is included in the F110W filter bandpass. In this calculation, we assume the F110W flux density as a pure continuum (contribution from $\mathrm{C}$ III] emission fluxes in the F128N filter to the F110W filter is less than $1 \%$ ). 
F110W

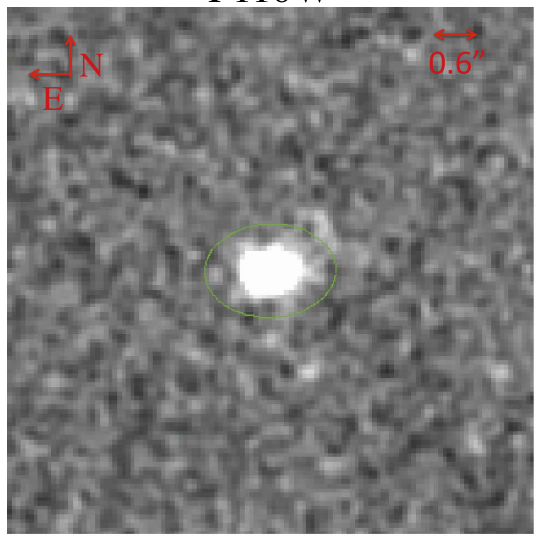

\section{F128N}

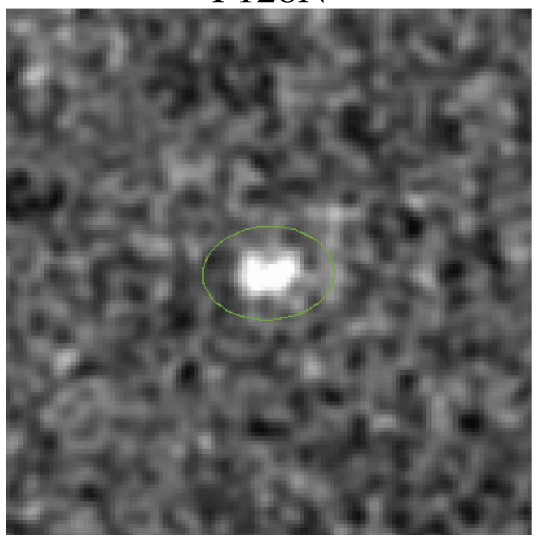

\section{F160W}
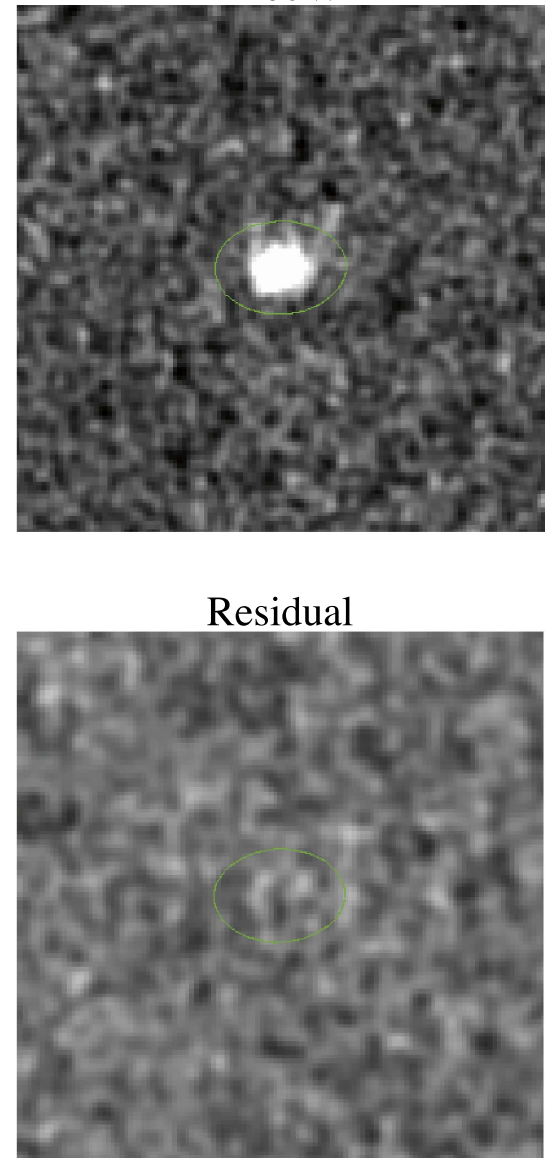

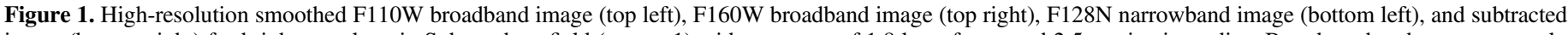

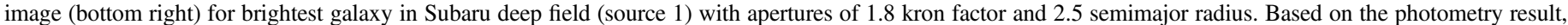

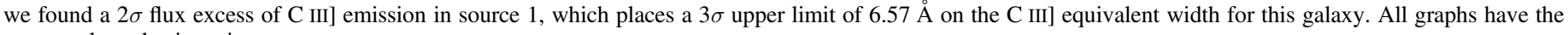
same scale and orientation.

After subtracting the continuum, we obtain the residual flux of the galaxy $F_{\mathrm{C} \text { III }}=3.34 \pm 1.81 \times 10^{-18} \mathrm{erg} \mathrm{s}^{-1} \mathrm{~cm}^{-2}$. The residual flux is calculated by $F_{\mathrm{C} \text { III }]}=\left(F_{\mathrm{F} 128 \mathrm{~N}}-F_{\mathrm{F} 125 \mathrm{~W}}\right) * \mathrm{FWHM}_{\mathrm{F} 128 \mathrm{~N}}$. There is a tentative $2 \sigma$ excess of flux measured in $\mathrm{F} 128 \mathrm{~N}$ compared to the continuum level. We use a $3 \sigma$ upper limit of $F_{\mathrm{C} \text { III }]} \leqslant 5.43 \times 10^{-18} \mathrm{erg} \mathrm{s}^{-1} \mathrm{~cm}^{-2}$, and we place a $3 \sigma$ upper limit on EW of $6.57 \AA$. We also examine the morphology of this galaxy. From the surface brightness profile in F110W imaging, this galaxy may contain two components. The continuumsubtracted image is shown in Figure 1.

The photometric results for the four galaxies in the SXDF are summarized in Table 1 . We do not detect C III] in any of those galaxies. Their $3 \sigma$ upper limits on C III] EWs range from 4.47 to $29.70 \AA$. Source 2 is the brightest galaxy in the SXDF field. We place a stringent $3 \sigma$ upper limit on the EW of $4.47 \AA$ and a $3 \sigma$ upper limit of flux of $3.36 \times 10^{-18} \mathrm{erg} \mathrm{s}^{-1} \mathrm{~cm}^{-2}$. Sources $3-5$ reside in the regions that are close to the detector edges. These regions have relatively higher noise levels that result in weaker constraints on $\mathrm{C}$ III] emission. For example, for source 5 , we place a $3 \sigma$ upper limit on the EW of $29.70 \AA$, much higher than that of source 2. Further, we stack all five galaxies in both the SDF and SXDF fields. ${ }^{8}$ We obtain a $3 \sigma$ upper limit

\footnotetext{
8 We stack the F128N and the F125W images using a weighted average (each source weighted by $\frac{1}{\sigma^{2}}$ after normalizing the fluxes). For source 1, we use the $\mathrm{F} 110 \mathrm{~W}$ and $\mathrm{F} 160 \mathrm{~W}$ images to get an averaged $\mathrm{F} 125 \mathrm{~W}$ image
}

of $4.20 \AA$ on the mean C III] EW and place a $3 \sigma$ upper limit of $F_{\text {stack }} \leqslant 2.55 \times 10^{-18} \mathrm{erg} \mathrm{s}^{-1} \mathrm{~cm}^{-2}$ on mean flux. In Table 2, we summarize the constraints of the $\mathrm{C}$ III] emission in our galaxy sample. For a comparison, we further list some previous measurements in galaxies at similar redshifts of $z \gtrsim 6$.

\section{Discussion}

We have examined the strength of $\mathrm{C}$ III] emission in a sample of five $z=5.70$ galaxies. We do not detect $\mathrm{C} \mathrm{III]}$ in the galaxies in our sample but report the measurement on $\mathrm{C}$ III] flux $\left(3.34 \pm 1.81 \times 10^{-18} \mathrm{erg} \mathrm{s}^{-1} \mathrm{~cm}^{-2}\right)$ for the brightest source in our sample. We place a $3 \sigma$ upper limit of $4.20 \AA$ on the stacked rest-frame $\mathrm{EW}$ for all the five galaxies in our sample. In the following discussion, we compare our sample galaxies with previous studies (e.g., Stark et al. 2014, 2015, 2017; Rigby et al. 2015) through two main aspects: (1) galaxy properties and (2) sample selection and depth of the survey.

\subsection{Galaxy Properties}

According to previous studies on C III] emitters (e.g., Stark et al. 2014, 2015, 2017; Rigby et al. 2015), several galaxy properties correlate with the strength of $\mathrm{C} \mathrm{III]} \mathrm{emission.} \mathrm{These}$ galaxy properties include galaxy metallicity, galaxy luminosity, Ly $\alpha$ line strength, stellar population, and the ionization parameter. Using our current data, we compare: (1) galaxy 
2
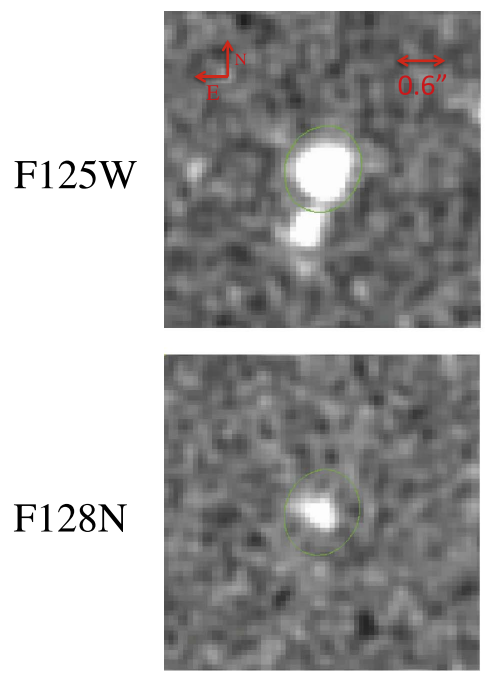

3
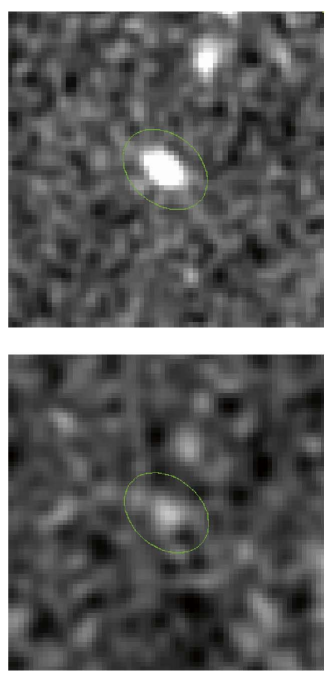

4
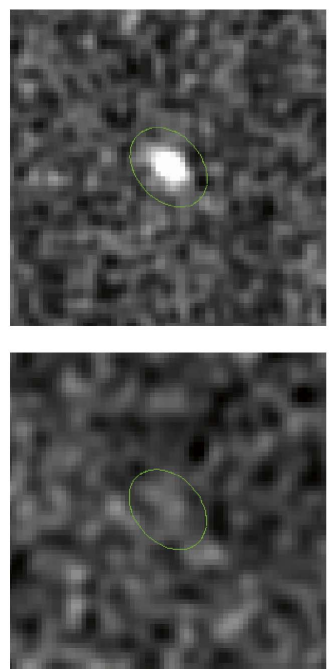

5
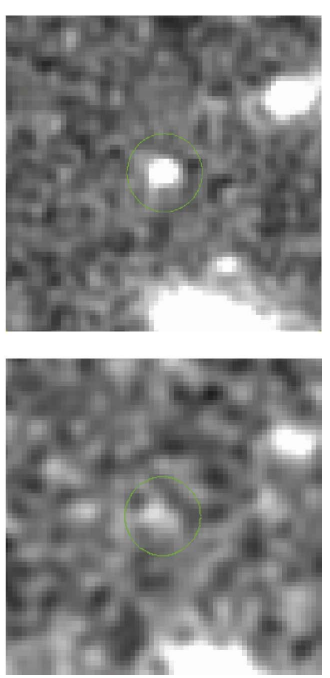

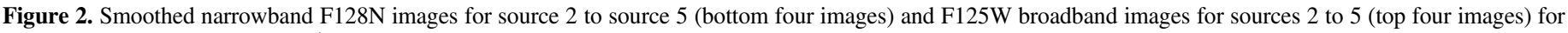

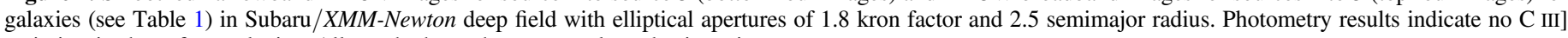
emission in these four galaxies. All graphs have the same scale and orientation.

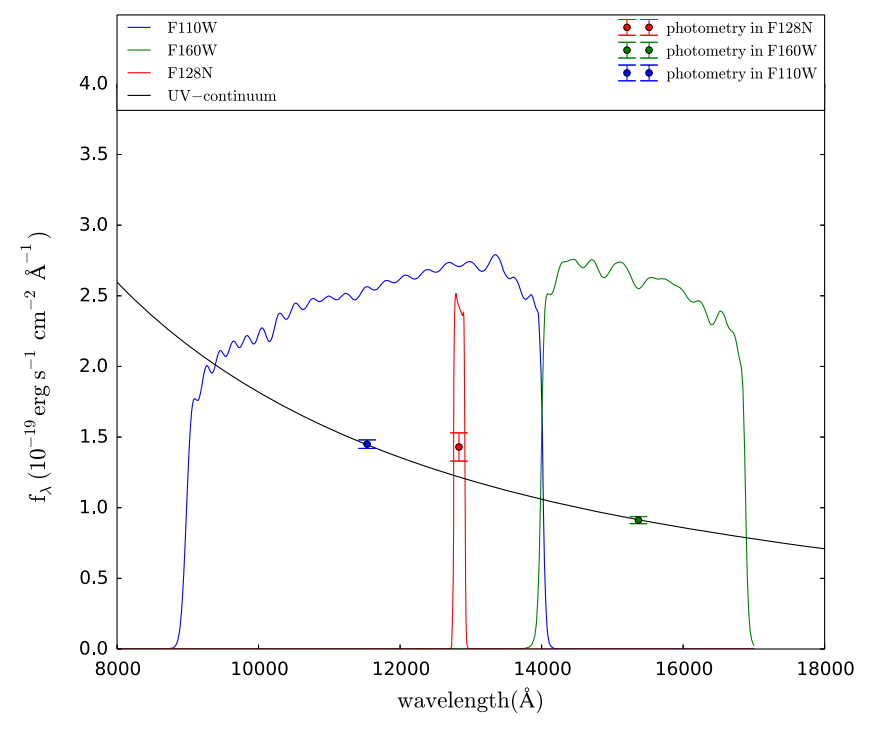

Figure 3. C III] emission and the best-fit spectrum (black line) with UVcontinuum fitted by photometry in WFC3/F110W (blue dot with error bar) and photometry in WFC3/F160W (green dot with error bar) of the brightest galaxy in our sample in SDF. The photometry in WFC3/F128N (red dot with error bar) shows a $2 \sigma$ excess of the WFC $3 / \mathrm{F} 128 \mathrm{~N}$ flux compared with the fitted UVcontinuum at the same wavelength. The filter response curves are plotted for WFC3/F110W filter (blue curve), WFC3/F160W (green curve) and WFC3/ F128N filter (red curve). The photometry data from F110W and F160W bands are from Jiang et al. (2013), while the photometry data from F128N are from our HST observation.

luminosity, (2) Ly $\alpha$ emission, and (3) metallicity to other galaxies having $\mathrm{C}$ III] detections or constraints in previous literature.

In Stark et al. (2014), intrinsically fainter galaxies tend to have stronger $\mathrm{C}$ III] emission at $z \sim 2$. Eleven out of 14 strong C III] emitters that have a rest-frame EW $>5 \AA$ and have a low intrinsic luminosity $\left(M_{\mathrm{UV}}>-19.30\right)$ in Stark et al. $(2014,2015,2017)$. The galaxies in our sample have a higher luminosity ( $M_{\mathrm{UV}}$ ranging from $\sim-20.48$ to -22.17 ). If galaxies at $z=5.7$ have similar properties with those in Stark et al. (2014) at $z \approx 2$, then we expect moderate C III] EWs $(<5 \AA$ ) for our galaxy sample.

Previous studies on C III] emission in luminous galaxies with $M_{\mathrm{UV}}<-20$ report relatively weaker C III] EWs. Rigby et al. (2015) study the C III] emission in a sample of galaxies with high luminosity $\left(M_{G}<-20\right)$. They find that a large fraction of $17 z \sim 2-4$ gravitational-lensed star-forming galaxies and 46 local galaxies have moderate C III] emission (EWs less than $5 \AA$ at rest frame). The composite spectrum of $z \sim 3$ LBGs with luminosity $M_{R} \sim-21$ in Shapley et al. (2003) and $z \sim 2.4$ star-forming galaxies with luminosity $M_{R} \sim-21$ in Steidel et al. (2016) also have EWs of C III] emissions $\sim 2 \AA$ at rest frame. Our galaxy sample has luminosities similar to that in Rigby et al. (2015), Shapley et al. (2003), and Steidel et al. (2016), and our C III] constraints are consistent with their results. Such consistency may be due to the similarity in galaxy luminosities.

A positive correlation between $\mathrm{C} \mathrm{III]} \mathrm{EW}$ and $\mathrm{Ly} \alpha \mathrm{EW}$ is suggested by Shapley et al. (2003) and is further illustrated in Stark et al. $(2014,2015)$ using galaxies at $z \sim 2-7$. Using the Ly $\alpha-\mathrm{C}$ III] correlation determined in these studies, we can estimate the expected C III] emission for our galaxy sample. For galaxy source 1 with a rest-frame Ly $\alpha$ EW of $21_{-2}^{+3} \AA$ (Shimasaku et al. 2006), the rest-frame C III] EW is expected to be $\approx 1.5 \AA$. The $3 \sigma$ upper limit on $\mathrm{C}$ III] EW of source 1 is $6.57 \AA$ (see Table 2), consistent with the correlation between Ly $\alpha$ and $\mathrm{C} \mathrm{III]} \mathrm{emission.} \mathrm{Using} \mathrm{this} \mathrm{C} \mathrm{III]-Ly} \alpha$ correlation, we can estimate the $\mathrm{C}$ III] strength for the other galaxies in the SXDF field (sources 2-5). The galaxies in the SXDF have Ly $\alpha$ EWs (rest-frame) ranging from $61.6_{-13.1}^{+21.8}$ to $106.4_{-40.3}^{+107.0} \AA$. If we assume the rest-frame Ly $\alpha$ EWs range from 61.6 to $106.4 \AA$ in these galaxies, the rest-frame $\mathrm{C}$ III] EWs are expected to be $\sim 4.0-8.0 \AA$. This expected EW range is lower than our current $3 \sigma$ upper limits on EWs in these sources (15.09-29.70 $\AA$ ). Thus, we conclude that our results are generally consistent with the Ly $\alpha-\mathrm{C}$ III] correlation found in previous studies (e.g., Shapley et al. 2003; Stark et al. 2014, 2015).

The C III] strength is also strongly affected by galaxy metallicity. Strong C III] emitters require a metal-poor 
Table 2

Properties of Galaxies in Our Work and Previous Work

\begin{tabular}{|c|c|c|c|c|c|c|}
\hline Source & $z_{\mathrm{Ly} \alpha}$ & $M_{\mathrm{UV}}$ & $\begin{array}{l}\operatorname{Ly} \alpha \mathrm{EW} \\
(\AA)\end{array}$ & $\begin{array}{c}\text { C III] EW } \\
(\AA)\end{array}$ & $\begin{array}{l}\text { C III] Flux or Upper Limits } \\
\left(10^{-18} \mathrm{erg} \mathrm{s}^{-1} \mathrm{~cm}^{-2}\right)\end{array}$ & References \\
\hline 1 & 5.70 & -22.17 & $21_{-2}^{+3}$ & $\leqslant 6.57(3 \sigma)$ & $\leqslant 5.43(3 \sigma)$ & this work, (1) \\
\hline 2 & 5.69 & -22.20 & $\cdots$ & $\leqslant 4.47(3 \sigma)$ & $\leqslant 3.36(3 \sigma)$ & this work, (2) \\
\hline 3 & 5.75 & -20.86 & $61.6_{-13.1}^{+21.8}$ & $\leqslant 15.09(3 \sigma)$ & $\leqslant 3.30(3 \sigma)$ & this work, (2), (3) \\
\hline 4 & 5.69 & -20.54 & $106.4_{-40.3}^{+107.0}$ & $\leqslant 19.95(3 \sigma)$ & $\leqslant 3.12(3 \sigma)$ & this work, (2), (3) \\
\hline 5 & 5.70 & -20.48 & $79.3_{-14.2}^{+22.1}$ & $\leqslant 29.70(3 \sigma)$ & $\leqslant 3.93(3 \sigma)$ & this work, (2), (3) \\
\hline Stacking & $\cdots$ & $\cdots$ & $\cdots$ & $\leqslant 4.20(3 \sigma)$ & $\leqslant \mathbf{2 . 5 5}(3 \sigma)$ & this work \\
\hline EGS-zs8-1 & 7.73 & -22.23 & $21 \pm 4$ & $22 \pm 2$ & $8.1 \pm 0.71$ & (4) \\
\hline EGS-zs8-2 & 7.48 & -22.08 & $9.3 \pm 1.4$ & $<7.1(3 \sigma)$ & $<2.3(3 \sigma)$ & (4) \\
\hline GN-108036 & 7.21 & -21.89 & 33 & $7.6 \pm 2.8$ & $2.2 \pm 0.8$ & (5) \\
\hline A383-5.2 & 6.03 & -21.57 & 138 & $22.5 \pm 7.1$ & $8.9 \pm 2.7$ & (5) \\
\hline A1689-zD1 & 7.50 & -22.11 & $\ldots$ & $\leqslant 4(3 \sigma)$ & $\leqslant 2(3 \sigma)$ & (6) \\
\hline
\end{tabular}

Note. Top five sources are galaxies in our work and bottom five sources are galaxies in Stark et al. $(2015,2017)$ and Watson et al. (2015). EW represents equivalent width (rest frame). All upper limits are $3 \sigma$ upper limits. $M_{\mathrm{UV}}$ for sources $2-5$ and bottom five sources is calculated by assuming a average $\mathrm{UV}$-slope $=-2.3$ found in a large sample of $z>6$ galaxies (Jiang et al. 2013).

References. (1) Shimasaku et al. (2006), (2) Ouchi et al. (2005), (3) Ouchi et al. (2008), (4) Stark et al. (2017), (5) Stark et al. (2015), (6) Watson et al. (2015).

interstellar medium with $Z \approx 0.04-0.13 Z_{\odot}$ (Stark et al. 2014). Various authors (e.g., Caldwell et al. 1992; Salzer et al. 2005; Savaglio et al. 2005; Guseva et al. 2009; Izotov et al. 2011, 2014; Zahid et al. 2011; Onodera et al. 2016) further suggest that there is a correlation between the galaxy luminosity and galaxy metallicity. Based on the luminosity and metallicity relation (L-Z relation) fitted by Zahid et al. (2011) at $z \sim 0.8$, the galaxy magnitudes of $M_{\mathrm{UV}}<-20.00$ correspond to metallicities of $12+\log (\mathrm{O} / \mathrm{H})>8.78 \pm 0.23$. Note the true errors will be much larger than 0.3 dex due to systematical uncertainties in this conversion, and the overall normalization in metallicity in Zahid et al. (2011) tends to bias the estimated metallicity to a larger value. We also need to take into account that galaxies of fixed luminosity will have lower metallicity at higher redshift. Through this conversion, the higher galaxy luminosities in our sample, with $M_{\mathrm{UV}}$ ranging from $\sim-20.48$ to -22.17 , may correspond to a significantly higher metallicity (e.g., $>0.1 Z_{\odot}$ ) compared with the strongly lensed galaxy sample in Stark et al. (2014, 2015) $\left(M_{\mathrm{UV}}>-19.30\right)$. Since $\left.\mathrm{C} \mathrm{III}\right]$ emission is sensitive to the metallicity (Stark et al. 2014), relatively higher metallicilty in our sample may yield lower EWs compared to galaxies in Stark et al. $(2014,2015)$.

\subsection{Sample Selection}

Stark et al. (2017) search for the C III] emission in a sample of two $z \geqslant 7$ luminous galaxies by using deep ground-based spectroscopy. From the photometry in rest-frame optical, these two galaxies are expected to have strong [O III] and $\mathrm{H} \beta$ emission. The spectroscopic observations on these two galaxies suggest that the galaxy at $z=7.73$ has a robust detection on C III] (Stark et al. 2017), with a C III] EW of $22 \pm 2 \AA$. The other galaxy has a $3 \sigma$ upper limit of $7.1 \AA$ on the C III] EW (Stark et al. 2017). The galaxy sample we selected is only based on their redshifts, without any consideration of the strength of [O III] and $\mathrm{H} \beta$ emission. Thus, compared with the extremely strong C III] emitters in Stark et al. (2017), our selection technique is different, which could be another reason for the moderate C III] EWs.

Also, our survey is slightly shallower than the extremely deep spectroscopy presented in Stark et al. (2015, 2017). The
$1 \sigma$ flux limit is $0.4 \times 10^{-18} \mathrm{erg} \mathrm{s}^{-1} \mathrm{~cm}^{-2}$ in Stark et al. (2015), smaller than our depth of $1 \sigma \sim 1 \times 10^{-18} \mathrm{erg} \mathrm{s}^{-1} \mathrm{~cm}^{-2}$. If we assume source 1 indeed has a C III] line flux of $3.34 \times 10^{-18} \mathrm{erg} \mathrm{s}^{-1} \mathrm{~cm}^{-2}$ (see Table 1), then we should be able to detect it with a $3 \sigma$ level with the depth in Stark et al. (2015). Deeper data may be needed to fully characterize the C III] emission in source 1 , the brightest LAE at $z=5.7$.

\section{Conclusions}

Using 12-orbit $H S T / \mathrm{F} 128 \mathrm{~N}$ narrowband imaging and 2-orbit $H S T / \mathrm{F} 125 \mathrm{~W}$ broadband imaging, we investigated the $\mathrm{C}$ III] emission in a sample of five galaxies in the SDF and the SXDF. We report a non-detection on $\mathrm{C}$ III] emission for the galaxies in our sample. Using the stacked image, we place a $3 \sigma$ upper limit of $4.20 \AA$ on the mean EW of five galaxies. Our photometric results suggest that the $\mathrm{C}$ III] emission may be moderate in a relatively high luminosity LAE sample at $z=5.7$, with galaxy magnitudes ranging from $\sim-20.48$ to -22.17 . Stark et al. (2017) suggest strong C III] emissions may also be found in galaxies with high intrinsic luminosity and low metallicity. Current samples on C III] candidates are biased toward luminous spectroscopically confirmed galaxies at $z>5.7$. Targeting galaxies with fainter intrinsic luminosities is needed in the future for fully understanding the rest-frame UV/optical emission lines in galaxies in or at the end of the reionization epoch. Also, in five candidates of $\mathrm{C}$ III] emitters of spectroscopically confirmed galaxies at $z>6$ (Stark et al. 2015, 2017; Watson et al. 2015), three of them are found to be C III] emitters with EWs ranging from $\sim 7.6$ to $22.5 \AA$ (Stark et al. 2015, 2017), while two of them are non-detections (Watson et al. 2015; Stark et al. 2017). The present studies (this work and Stark et al. 2015, 2017; Watson et al. 2015) on C III] emission suggest that the $\mathrm{C}$ III] EWs at $z>5.70$ have a wide range of distribution. Future facilities, including Giant Segmented Mirror Telescopes and the James Webb Space Telescope, will thoroughly probe the $\mathrm{C} \mathrm{III}$ ] emission in a much fainter high- $z$ galaxy population and measure other rest-frame $\mathrm{UV} /$ optical emission lines to fully characterize the properties of the typical galaxies at the reionization epoch. 
We thank the support from NASA through grant HST-GO13644 from the Space Telescope Science Institute. Support for part of this work was also provided by NASA through the Hubble Fellowship grant HST-HF2-51370 awarded by the Space Telescope Science Institute, which is operated by the Association of Universities for Research in Astronomy, Inc., for NASA, under contract NAS 5-26555. D.P.S. acknowledges support from the National Science Foundation through the grant AST-1410155.

\section{References}

Bertin, E., \& Arnouts, S. 1996, A\&AS, 117, 393

Bouwens, R. J., Illingworth, G. D., Oesch, P. A., et al. 2015, ApJ, 803, 34

Cai, Z., Fan, X., Jiang, L., et al. 2011, ApJL, 736, L28

Cai, Z., Fan, X., Jiang, L., et al. 2015, ApJL, 799, L19

Caldwell, N., Armandroff, T. E., Seitzer, P., \& Da Costa, G. S. 1992, AJ, 103,840

Caruana, J., Bunker, A. J., Wilkins, S. M., et al. 2014, MNRAS, 443, 2831

Choudhury, T. R., Puchwein, E., Haehnelt, M. G., \& Bolton, J. S. 2015, MNRAS, 452, 261

Ellis, R. S., McLure, R. J., Dunlop, J. S., et al. 2013, ApJL, 763, L7

Erb, D. K., Pettini, M., Shapley, A. E., et al. 2010, ApJ, 719, 1168

Erb, D. K., Steidel, C. C., Trainor, R. F., et al. 2014, ApJ, 795, 33

Finkelstein, S. L., Ryan, R. E., Jr., Papovich, C., et al. 2015, ApJ, 810, 71

Guseva, N. G., Papaderos, P., Meyer, H. T., Izotov, Y. I., \& Fricke, K. J. 2009, A\&A, 505, 63

Izotov, Y. I., Guseva, N. G., Fricke, K. J., \& Henkel, C. 2014, A\&A, 561, A33
Izotov, Y. I., Guseva, N. G., \& Thuan, T. X. 2011, ApJ, 728, 161

Jaskot, A. E., \& Ravindranath, S. 2016, ApJ, 833, 136

Jiang, L., Egami, E., Mechtley, M., et al. 2013, ApJ, 772, 99

Kashikawa, N., Shimasaku, K., Matsuda, Y., et al. 2011, ApJ, 734, 119

Koekemoer, A. M., Fruchter, A. S., Hook, R. N., \& Hack, W. 2003, in The 2002 HST Calibration Workshop: Hubble after the Installation of the ACS and the NICMOS Cooling System, ed. S. Arribas, A. Koekemoer, \& B. Whitmore (Baltimore, MD: STScI), 337

McLure, R. J., Dunlop, J. S., Bowler, R. A. A., et al. 2013, MNRAS, 432 2696

Mesinger, A., Aykutalp, A., Vanzella, E., et al. 2015, MNRAS, 446, 566

Onodera, M., Carollo, C. M., Lilly, S., et al. 2016, ApJ, 822, 42

Ouchi, M., Shimasaku, K., Akiyama, M., et al. 2005, ApJL, 620, L1

Ouchi, M., Shimasaku, K., Akiyama, M., et al. 2008, ApJS, 176, 301

Planck Collaboration, Ade, P. A. R., Aghanim, N., et al. 2016, A\&A, 594, A13

Rigby, J. R., Bayliss, M. B., Gladders, M. D., et al. 2015, ApJL, 814, L6

Salzer, J. J., Lee, J. C., Melbourne, J., et al. 2005, ApJ, 624, 661

Savaglio, S., Glazebrook, K., Le Borgne, D., et al. 2005, ApJ, 635, 260

Shapley, A. E., Steidel, C. C., Pettini, M., \& Adelberger, K. L. 2003, ApJ, 588,65

Shimasaku, K., Kashikawa, N., Doi, M., et al. 2006, PASJ, 58, 313

Stark, D. P. 2016, ARA\&A, 54, 761

Stark, D. P., Ellis, R. S., Charlot, S., et al. 2017, MNRAS, 464, 469

Stark, D. P., Richard, J., Charlot, S., et al. 2015, MNRAS, 450, 1846

Stark, D. P., Richard, J., Siana, B., et al. 2014, MNRAS, 445, 3200

Steidel, C. C., Strom, A. L., Pettini, M., et al. 2016, ApJ, 826, 159

Watson, D., Christensen, L., Knudsen, K. K., et al. 2015, Natur, 519, 327

Zahid, H. J., Kewley, L. J., \& Bresolin, F. 2011, ApJ, 730, 137

Zitrin, A., Ellis, R. S., Belli, S., \& Stark, D. P. 2015, ApJL, 805, L7 